\title{
A LOOK INTO THE FUTURE OF THE ONLINE PLATFORM
}

\author{
Mrinal Paliwal \\ SOEIT, Sanskriti University, Mathura, Uttar Pradesh, India
}

\begin{tabular}{|c|c|}
\hline Journal & $\begin{array}{l}\text { Samvakti Journal of Research in Information Technology } \\
\text { https://www.sjrit.samvaktijournals.com } \\
\text { Volume } 2 \text { Year of Volume } 2021 \text { Page No : } 83-91\end{array}$ \\
\hline Discipline & E-Commerce \\
\hline Conference & $\begin{array}{l}\text { A virtual international conference on redefining and transforming the role of higher } \\
\text { education in sustainable development }\end{array}$ \\
\hline Conference & Start Date: September 30, 2021 \\
\hline Dates & End Date : September 30, 2021 \\
\hline Institute Name & $\begin{array}{l}\text { JAIN (Deemed-to-be University) in association with Council for Industrial } \\
\text { Innovation and Research }\end{array}$ \\
\hline $\begin{array}{l}\text { Date Received } \\
\text { ID } \\
\text { Dol }\end{array}$ & $\begin{array}{ll}\text { Publication Date } & \text { : December 11, } 2021 \\
\text { Paper Type } & : \text { Conference Paper } \\
\text { Dol URL } & : \text { https://dx.doi.org/10.46402/2021.02.20 }\end{array}$ \\
\hline
\end{tabular}

Access Type : Open Access (Attribution-NonCommercial-NoDerivatives 4.0 International) (C) 2021 Mrinal Paliwal with publication rights granted to Samvakti Journals

\section{ABSTRACT}

The internet has changed the world of computers and communication, like nothing else. Telegraphs, telephones, radios and computers all laid the scene for the incomparable convergence of Internet capabilities. The Internet is a worldwide broadcasting capacity, an information distribution system and the method by which people and their computers communicate and interact, regardless of their location. The Internet is also one of the most prominent instances of ongoing IT investment and commitment to research and development. The government, business and academics have worked together to develop and implement this fascinating new technology, starting with early research on packet change. The Internet is now a wide-ranging information infrastructure, the first iteration of the national information infrastructure. Its history, encompassing technology, organization and culture, is complex and embraces many aspects. And as we go toward increasing usage of online resources in order to accomplish e-commerce, acquisition of information and community activities, its effects affect not only computer technology but society as a whole. 
KEYWORDS: ARPANET, Commercialization, Communication, Internet, RFC.

\section{INTRODUCTION}

One of the earliest documented explanations of the social relationships that might be facilitated via networking was provided by J.C.R. Harvard University of Technology's Licklider in a series of memos sent in july 1962, detailing his idea of a "Galaxy's Connection". Licklider imagined a network of computers that were all linked to one another and from which data and programmes from all over the world could be accessed simply by anybody. In essence, the idea were more similar to the web of now. Leonard Kleinrock of the Massachusetts Institute of Technology (MIT) released the 1st research publication on the theory of June 1961 was the first time package shifting was used. When Kleinrock was a teenager, he convinced Roberts that utilizing packets rather than circuits might be a viable option for communication. This constituted a significant milestone forward in the development of networked computers. The second important strategy was to force each other to communicate with the machines ${ }^{[1]}$.

When collaborating alongside Tom Fenner in 1965, on an investigation of this idea, The TX-2 supercomputer in Boston was linked to the Q-32 by Robbins. computer via a reduced keypad connection in Californian connection, thus establishing 1st computing system that spans a large region in history. In the end, the investigation provided evidence because moment machines have the potential to operate effectively together, executing programmes and obtaining data from distant machines as needed, but that the telephone equipment disconnected from the circuit were completely insufficient for that the task. Kleinrock's opinion about this requirement of routing protocol was invented thus reinforced.

The ARPANET was Robbins' suggestion, who DARPA was visited in late 1966 to improve the concept of a connection of computers. He worked fast to put up a proposal for the network, which was published in 1967. The ARPANET switches (also known as IMPs) were constructed by Bolt, Beranek and Newman Corporation (BBN) inside the direction of Frank Heart, with Robert Kahn in charge of the whole mechanism architecture. Herman Franks and its associates Robin collaborated together friends at Networks Research Incorporated to develop improve the structure and the economy of the network, and the results were impressive. In part, this was owing to Kleinrock's early application concept of frame relay at UCLA, as well as his main emphasis research, Measuring and architecture His UCLA Networking Measurements Centre were chosen as the ARPANET's initial port. All of BBN brought it altogether in November 1969. built UCLA's very 1 st change and linked it to the 1 st machine to serve as the server, marking the beginning of the Information Age ${ }^{[2]}$. 
During the month of The Networks Study Team was formed in November 1970. under the leadership of Roach, Steven developed the first organise ARPANET protocol, known as NCP stands for Wireless Protocols Designed. The ARPANET websites are similar to the ARPANET websites. finished the implementation of the Network Control Protocol (NCP), Members of the system were allowed to start building apps. over the years 1971-1972. A major and effective ARPANET presentation took place in September 1972. took conducted, marking the first public demonstration of this revolutionary technologies for networks.

In 1972, the first the use of digital email programme, known as the "hot" programme, were revealed. When BBN's Danny Murphy was motivated by the need for a fast coordination mechanism for ARPANET engineers in March, he developed the program that sends or receives e mails in their most basic form that you see here. In the years that followed, email grew in popularity as the more important of ten used network programme and as a forerunner depending on the type of communications process between individuals that we see today on the internet ${ }^{[3]}$.

The Area Networks Protocols is a method for managing networks is the name of the standard administration system presently in use on the Internet, and it is its primary building component (SNMP). Later as in 1980s, it was developed, and it is extensively networking gadgets' compatibility today. It is possible the capacity to comprehend \& express basic typed variables using the SNMP management protocol, which is a specialpurpose management protocol. Known as an agent, the software component responsible for handling the related Attempts to got totally and gaining access to inner information structures on gadgets that can be manipulated is a software component. Additionally, an agent may create notifications under specific conditions and, in additional to generating these queries, transmit these as unwanted emails to the administration software and generating notifications (manager). The manager-agent paradigm is the name given to this design.

Components of the managerial data base, that are developed in a programming languages known as Data Managerial Architecture, for handling tangible database schemas particular procedures or technology created and standardized (SMI). Smm is an information programming languages built on Abstraction Syntax Notation 1 standard (ASN.1). It necessitates the normalization of complicated layered data structures into a collection of interconnected MIB records that are theoretical. The notion of structured data types, objects, and methods are not presently supported by the system. Despite the fact that The SNMP protocol is currently widely used. known and extensively implemented, It always is the case. primarily used to manage networking gadgets as well as is seldom used to manage mechanisms. Although SNMP is widely used in There are several types 
of system component control. many location relevant regions it has only played a small role thus far ${ }^{[4]}$.

Due to the fact that SNMP technologies is only utilized in a limited the amount of managers domains, it is not unexpected that other techniques had being developed suggested in recent years. This article examines these ideas and summarizes the outcomes of relevant debates that happened inside the Internet Joint Committee on Engineers, the Working Group on Web Studies, and the Council of the Web Infrastructure in recent months. The remainder of this article is arranged in the following manner. As a starting point, we will discuss the mismatch between the needs of Internet network operators and the evolution of the SNMP protocol since its creation. Following that, we'll go through several evolutionary methods to improving the SNMP framework. Finally, we look at some of the most innovative methods that are Webhosts with the Extensible Annotation Language.

\section{The Fundamentals of Internet Technology:}

In the beginning, the ARPANET was built on the premise that there would be many independent networks of a somewhat random character, which eventually evolved into the Internet. Following that, it quickly extended to encompass Additional systems include particle space systems, floor particle radio channels, and others, beginning with ARPANET, which was the first packet-switching network to be deployed. The Internet today is a manifestation of a basic it is a technological concept widely accepted: accessible networking ${ }^{[5]}$.

Every option is available in that strategy. specific connection infrastructure is never governed by a specific nevertheless, networking is designed is instead left to the discretion of the service provider and accomplished via the creation of a morpho "intended to supplement infrastructure" allows the provider to connect to other networks. It is possible to customize each network to meet the needs of a certain environment and set of end users. Developed early 1972, by Khan, soon after his arrival at DARPA, the concept of open-architecture networking was guided by four fundamental principles:

1. It was necessary for each independent network to be self-contained, and it was not possible for any such network to be improved on the inside until it was connected to the web.

2. Interactions will be conducted on the premise of a finest premise If a package is missing, does not reach it to its ultimate location, it may be simply rebroadcast from the origin.

3. In order to connect the networks, black boxes will be needed (later called gateways and routers). The gateways will not store any information about individual packet flows something passes into them and transforms they're straightforward to manage 
as well as preventing complex Resilience to and rehabilitation after a variety of setbacks scenarios.

4. When it comes to organizational development, there will be no global impact.

\section{The monetization of recent Internet:}

The Web's industrialization has never engaged just the creation of cheap a personal connection networks, and likewise the commercialization of Internet technology goods and services. Earlier inside the 1980s, many of manufacturers started TCP/IP is being incorporated into their solutions due recognized a market for this networking method among their customers. Unfortunately, they lacked solid proof on how the technology was meant to function and how their customers were anticipated to utilize the service.

A three-day course for all vendors was arranged by Daniel Lynch in collaboration with the Internet Architecture Board (IAB) in 1985 to teach them whatever TCP/IP was \& why it works and could not accomplish effectively, recognizing the lack of accessible proper education and knowledge at this time. The majority of the presenters were from DARPA's research lab, where these procedures were created and are now in use in the field on a daily basis. A total of about 250 vendor representatives addressed a group of 50 innovators and experimenters ${ }^{[6]}$.

The inaugural Interop trade exhibition, held in September 1988 and attended by 50 businesses and 5,000 engineers from prospective client groups, showed interoperability across vendor technologies. Since then, Interop has grown tremendously, and today it is a yearly event with an There were greater then 250,000 people in the crowd. People who come to learn about these items are effective together what additional items do you have or what 's new technologies in seven locations around the world. Interop is performed in 7 cities across the globe.

Recent years have seen an increase in commercialization activity on an unprecedented scale. Market efforts were first concentrated mostly on makers of basic networking devices and service providers that provided access to the Internet and basic Internet services. As a "commodity" service, the Internet has nearly reached the status of a commodity, and the application of this international data architecture as a foundation for additional business solutions has recently received a great lot of attention.

As a result of the broad and fast adoption of browsers and web-based technologies, this practice has increased, providing users with quick access to connected information from across the world. These products for the finding, transfer, and retrieval of this information are already available, and many of the most recent technologies are geared at providing ever-more complex In addition of fundamental Web data, there are informational solutions connections $^{[7]}$. 


\section{Role of Documentation:}

The availability of free and open access to fundamental information, particularly protocol specifications, has been a significant factor in the fast development of the Internet. The Dialup modems \& the web's origins aided in the development of the legacy of openness in academia publishing of concepts \& outcomes in the context of university research culture. The typical time of traditional academic publishing, on the other hand, were excessive regimented as well as sluggish for the kind of dynamic interchange of ideas that was needed for network building. S. made a significant decision in 1969. Crocker intended for the sequence of remarks on a request for comments to serve as an informal, quick means of disseminating ideas among network researchers ${ }^{[8]}$.

Crocker intended for the series of notes to serve as a casual, quick method of communication disseminating networking scientists' thoughts. In the beginning, the Image can be viewed was copied and distributed. sent via the postal service. As with the FTP protocols, became more widely used, the RFCs were produced as online files that could be viewed through FTP. RFCs are now readily available via the Web on hundreds of sites all around the globe, thanks to the Internet. SRI, in its capacity as the Network Information Center, was in charge of maintaining the online directories. For the required number of protocol assignments, Jon Postel worked as an as much as an RFC writer a management centralised manager, roles that he has maintained to this day.

\section{DISCUSSION}

The web were created during the period however there is a lot of moment it has persisted customer and friend computers, and networked networking into the age of desktop laptops, customer, and friend informatics computers, among other things. Even though LANs were not yet available, it was intended to support them in addition more modern Atm as well as framework -based services. However, the intention has changed. It was designed to serve a variety of tasks, ranging Data transfer, virtual domain, exchanging assets, and teamwork are just a few of the features available., and it was the forerunner of email and the World Wide Web in more recent years.

But, perhaps most significantly, It began as the work a tiny business group of committed individuals academics and has developed into a monetary achievement as a result of billions of dollars in yearly investments. If we believe that the Internet has everything, we are making a big mistake! The Internet, as opposed to the traditional networks of the telephone and television companies, is a machine creature. Indeed, it will continue to develop at the same pace as the computer industry in order to maintain its relevance in the future. 
The supply of new technologies such as well as true transportation, which may handle Channels of sound / picture, for example, is now in the process of developing. The accessibility to ubiquitous connection, i.e., the web itself, as well as efficient, cheap computing and portable communications, has enabled the emergence of a novel computer and networking model for the nomadic, which is described below ${ }^{[9]}$.

In the future, this development will offer us new games, Telephony over the web service, and web television service, among other things. In addition, it will enable greater sophistication kinds of reimbursement and price, which will undoubtedly become necessary in this world of commerce despite the pain associated with doing so. Everything from broadband home connection to satellites is in the process of changing in order to handle New iteration of core network technology has arrived, this time with varying features and specifications.

New access modes and service kinds will spur the development of additional submissions, that will lead to spur the development of the internet as a whole. Not how technology may advance, but how to deal with the process of change and growth itself, is the most urgent question for the Internet's long-term viability and success. While a core group of designers has always been at the forefront of Internet architecture, as a result of the increase in the quantity of outsiders engaged is expanded, composition of that group has changed.

The way we live has changed as a result of technological advancements, particularly in our data-driven environment. This is due in part to advances in semiconductors and communications technologies, which allow a wide range of gadgets to be connected across a system, providing us with techniques to connect and communicate among computers and people. The Internet-of-Everything, which includes the Web (IoT), Web (IoMT), Web (IoBT), Internet-of-Vehicles (loV), and so on, is another term for this phenomenon.

Anonymity and safety are two of several important concerns given the ubiquitousness of such devices in our society. In 2014, for example, it was found that over 750,000 customer devices had been hijacked to transmit phishing and spam messages. Ensuring the safety of the data, networks, and devices, as well as the secrecy of the information and information calculations, is critical in data-sensitive applications like loMT and IoBT. A threat to a system, on the other hand, might be the result of a poorly thought-out security solution.

For instance, in a normal In a municipal or army clinic, the Info Technologies (IT) team is normally in charge of the entire networking, comprising edge devices and loMT devices. It's unrealistic to expect IT employees to be familiar with every single connected device, 
even if they have systems administration permission to install upgrades and remote monitor the machine and its information, and so on ${ }^{[10]}$.

\section{CONCLUSION}

Due to the widespread popularity of the Internet, an unprecedented number of parties have been involved in the network, each having a vested both from a monetary and a philosophical standpoint interest the internet. Consider, Take, for illustration, the battle to establish the following informal gathering framework to govern the web, which can be seen in the arguments over domain namespace control, as well as the form of nextgeneration Internet Protocol addresses (IPv6 addresses). The framework, on the other hand, is increasingly challenging to characterise because of because of the huge quantity players. The business is also trying to establish the financial justification for the massive investments needed for future development, such as increasing home access to more suitable technology, which would be required to increase residential access to more appropriate technology, for example. If the Internet fails, This would never be the case since of a lack of technology, vision, or creativity, but rather because we have failed to chart a path and march together ahead of time. 


\section{REFERENCES}

[1] H. Schaffers, N. Komninos, M. Pallot, B. Trousse, M. Nilsson, and A. Oliveira, "Smart cities and the future internet: Towards cooperation frameworks for open innovation," Lect. Notes Comput. Sci. (including Subser. Lect. Notes Artif. Intell. Lect. Notes Bioinformatics), 2011, doi: 10.1007/978-3-642-20898-0_31.

[2] H. Rahman and R. Rahmani, "Enabling distributed intelligence assisted Future Internet of Things Controller (FITC)," Appl. Comput. Informatics, 2018, doi: 10.1016/j.aci.2017.05.001.

[3] V. Issarny et al., "Service-oriented middleware for the Future Internet: State of the art and research directions," J. Internet Serv. Appl., 2011, doi: 10.1007/s13174-0110021-3.

[4] C. Granell et al., "Future Internet technologies for environmental applications," Environ. Model. Softw., 2016, doi: 10.1016/j.envsoft.2015.12.015.

[5] J. M. Hernández-Muñoz et al., "Smart cities at the forefront of the future internet," Lect. Notes Comput. Sci. (including Subser. Lect. Notes Artif. Intell. Lect. Notes Bioinformatics), 2011, doi: 10.1007/978-3-642-20898-0_32.

[6] C. W. Tsai, C. F. Lai, and A. V. Vasilakos, "Future Internet of Things: open issues and challenges," Wirel. Networks, 2014, doi: 10.1007/s11276-014-0731-0.

[7] A. Kaloxylos et al., "Farm management systems and the Future Internet era," Comput. Electron. Agric., 2012, doi: 10.1016/j.compag.2012.09.002.

[8] W. Ding, Z. Yan, and R. H. Deng, "A Survey on Future Internet Security Architectures," IEEE Access. 2016, doi: 10.1109/ACCESS.2016.2596705.

[9] X. Liu, M. Zhao, S. Li, F. Zhang, and W. Trappe, "A security framework for the internet of things in the future internet architecture," Futur. Internet, 2017, doi: 10.3390/fi9030027.

[10] A. M. Oostveen et al., "Cross-disciplinary lessons for the future internet," Lect. Notes Comput. Sci. (including Subser. Lect. Notes Artif. Intell. Lect. Notes Bioinformatics), 2012, doi: 10.1007/978-3-642-30241-1_5.

$\underline{\underline{E N D}}$

Page No. 91 